E1 uso de efectivo y tarjetas débito como instrumentos de pago en Colombia

Constanza Martínez 
Lecturas de Economía, 90 (enero-junio 2019), pp. 71-95

Constanza Martínez.

El uso de efectivo y tarjetas débito como instrumentos de pago en Colombia

Resumen: Este documento estudia el impacto que la popularización de las tarjetas débito ha tenido sobre las preferencias de pago de los clientes bancarios con cuentas de depósito en Colombia. Con este fin, se utilizan modelos de ecuaciones simultáneas para analizar los pagos habituales y las compras al por menor de bienes no durables que este grupo poblacional realiza de manera presencial, en función de variables macroeconómicas, de infraestructura de pagos e idiosincráticas. Los resultados resaltan la relevancia de la tasa de interés de corto plazo, la infraestructura de pagos y el gravamen a los movimientos financieros en las preferencias por instrumentos de pago de dicha población. En particular, se observa que el aumento en el número de datáfonos ha comenzado a representar una amenaza a la preeminencia del efectivo; no obstante, factores idiosincráticos como la inseguridad y la informalidad incentivan su uso.

Palabras clave: demanda por dinero, tarjeta débito, costos transaccionales.

Clasificación JEL: E41, G21, D23.

The use of cash and debit cards as payment instruments in Colombia

Abstract: This paper studies the impact that the popularisation of debit cards has had on the payment preferences of retail banking clients with deposit accounts in Colombia. To this end, simultaneous equation models are used to analyse the usual payments and retail purchases of non-durable goods that these people execute in person, as a function of macroeconomic variables, the payments infrastructure, and idiosyncratic variables. The results underline the relevance of the short-term interest rate, the payments infrastructure, and the financial transaction tax on the preferences for payment instruments of this population. Particularly, it is observed that the rise in the number of point-of-sale devices bas started to represent a threat to the prevalence of cash, but that idiosyncratic factors such as insecurity and informality incentivise its usage.

Keywords: money demand, debit card, transaction costs.

JEL Classification: E41, G21, D23.

\section{L'utilisation des espèces et des cartes de débit en tant qu'instruments de paiement en Colombie}

Résumé: Cet article étudie l'impact de l'utilisation des cartes de débit sur les préférences de paiement des individus dotés d'un compte bancaire en Colombie. À cette fin, des modèles d'équations simultanées sont utilisés pour analyser les paiements et les achats au détail de biens non durables que ce groupe d'individus effectue directement chez les commergants, en fonction de variables macroéconomiques, de l'infrastructure de paiement et des paramètres mesurant l'idiosyncrasie des individus. Les résultats mettent en évidence que les préférences pour les instruments de paiement sont déterminées par le taux d'intérêt à court terme, l'infrastructure de paiement et la taxe sur les mouvements financiers. On constate en particulier que l'augmentation du nombre de terminaux de paiements électroniques a commencé à représenter une menace pour la prééminence de l'argent liquide. Cependant, les facteurs idiosyncratiques tels que l'insécurité et linformalité encouragent son utilisation.

Mots-clés: demande de monnaie, carte de débit, coûts de transaction.

Classification JEL: E41, G21, D2. 


\title{
El uso de efectivo y tarjetas débito como instrumentos de pago en Colombia
}

\author{
Constanza Martínez (iD ${ }^{\mathrm{a}}$ \\ -Introducción. -I. Preferencias de pago del consumidor: una revisión de literatura. -II. Pagos \\ con efectivo y tarjetas débito: un enfoque de flujo. -III. Datos. -IV. Resultados. \\ -Conclusiones. -Referencias.
}

doi: 10.17533/udea.le.n90a03

Primera versión recibida el 22 de noviembre de 2017; versión final aceptada el 13 de marzo de 2018

\section{Introducción}

Tradicionalmente, se ha hecho alusión a los medios de pago e instrumentos de pago como términos equivalentes que hacen referencia a la forma en la cual los consumidores pagan sus transacciones; sin embargo, estos términos cumplen funciones muy distintas. Según Hernández (2009), los medios de pago (efectivo, cuentas de depósito y crédito), por ser reserva de valor, sirven como unidad de cuenta, y permiten la compra de bienes y la acumulación de poder de compra; mientras que los instrumentos de pago (efectivo, cheque y tarjetas de crédito y débito) son los artefactos mediante los cuales se transfieren las órdenes de pago. Es decir, los medios de pago satisfacen las órdenes de transferencia de fondos que las personas emiten a través de instrumentos de pago. Para las tarjetas, el cheque y las transferencias de fondos, los medios de pago son las cuentas de depósito. Para la tarjeta de crédito y el efectivo, los medios de pago son el crédito y el efectivo, respectivamente. Los

a Constanza Martinez Ventura: profesional líder del Departamento de Seguimiento a la Infraestructura Financiera, Banco de la República, Colombia. Dirección postal: carrera 7 \#14-78. Dirección electrónica: amartive@banrep.gov.co.

https://orcid.org/0000-0001-6128-5095

Se agradecen las sugerencias de Clara Machado, Carlos León, Freddy Cepeda, Carlos Arango, Enrique Lopez y dos evaluadores anónimos, así como el apoyo de Aura María Ciceri en la consecución de los datos. Las opiniones expresadas en este documento son responsabilidad de la autora y, por tanto, no comprometen al Banco de la República ni a su Junta Directiva. 
Martínez: El uso de efectivo y tarjetas débito como instrumentos de pago en Colombia

instrumentos de pago distintos al efectivo son provistos por los bancos, y su uso requiere de una infraestructura de pagos física y tecnológica compuesta por canales transaccionales que facilitan el acceso a los servicios financieros. Estos canales están compuestos por oficinas bancarias, cajeros automáticos (ATM), datáfonos, audio servicios e internet (Hernández, 2009) ${ }^{1}$.

Si el efectivo es sucio, pesado y obsoleto, como lo afirman Drehmann, Goodhart y Krueger (2002), ¿por qué continúa siendo el instrumento de pago que más utilizan las personas en sus transacciones? Al parecer, las tecnologías de pago modernas tienen un bajo impacto sobre la preeminencia del efectivo debido a las bondades que el uso de este representa. La literatura de pagos (Humphrey et al., 1996b; Drehmann et al., 2002; Rambure \& Nacamuli, 2008; Arango \& Taylor, 2009) ha señalado, entre las ventajas del uso de efectivo, que: puede ser reutilizado inmediatamente, permite completar otras transacciones (divisible), elimina el riesgo de fraude (clonación de tarjetas bancarias), impide identificar a los participantes de la transacción (no es trazable) y es más aceptado que otros instrumentos de pago. En contraposición, existe un conjunto de factores por los cuales el efectivo no es usado en todo tipo de transacción, como: los pagos de alto valor, la distancia entre comprador y vendedor, los problemas de seguridad, los intereses que se dejan de percibir por usar efectivo (costo de oportunidad) y los períodos de gracia e incentivos ofrecidos a los usuarios de tarjetas de crédito (Rambure \& Nacamuli, 2008; Arango, Huynh \& Sabetti, 2011).

En Colombia, la introducción de tarjetas débito y ATM se dio a finales de 1970, pero su uso se popularizó a finales de 1990, con el aumento en el número de ATM y el ingreso de datáfonos al sector comercial. Por esta razón, es natural preguntarse: ¿cuál es el impacto que han tenido las tecnologías de pago electrónico en las preferencias de pago de la población?, ¿continúa el efectivo siendo el instrumento de pago más utilizado por la población colombiana? Este documento pretende responder a estos interrogantes considerando la demanda transaccional de dinero y dejando de lado los motivos de precaución y especulación, por tratarse de enfoques relacionados con teorías

1 En Colombia los dispositivos de pago electrónico se conocen como datáfonos, pero en otros países se conocen como equipos-TPV (terminales punto de venta), terminal de pago electrónico, terminal de pago PIN Pad o lector de tarjetas débito y crédito. 
de portafolio. En particular, se consideran los pagos habituales y las compras al por menor de bienes no durables que las personas realizan de manera presencial. La unidad de análisis son los clientes bancarios que tienen cuentas de depósito (corrientes y de ahorro), por ser los únicos que pueden decidir pagar sus compras con efectivo, tarjetas débito o cheques. No se consideran las tarjetas de crédito y los cheques rotativos porque dependen de los cupos de crédito asignados a los clientes y no de los depósitos bancarios. Tampoco se consideran la transferencia electrónica de fondos porque no se usa en compras y pagos presenciales al por menor. En este análisis se sigue un modelo similar al propuesto por Amromin y Chakravorti (2007), pues se incluyen las innovaciones financieras (canales transaccionales), pero, a diferencia de este, se usa una medida de flujo de efectivo y se estudia la determinación conjunta de los parámetros que afectan las decisiones de los agentes mediante modelos de ecuaciones simultáneas.

\section{Preferencias de pago del consumidor: una revisión de literatura}

En la literatura sobre pagos al por menor se han señalado las numerosas bondades de usar efectivo, entre las cuales el valor de la transacción es un elemento esencial para entender las decisiones de la población. De acuerdo con Snellman, Vesala y Humphrey (2001), Klee (2008) y Arango et al. (2011), los consumidores prefieren pagar sus transacciones de altas cuantías (i.e. compra de bienes durables) con transferencias electrónicas de fondos, cheques y tarjetas de crédito y débito, mientras que en las de cuantías bajas usan efectivo. Este último resultado se evidencia, incluso, en países desarrollados como Canadá, Australia, Francia y Países Bajos, pero existen casos como el de Alemania y Austria, en donde la preferencia por el uso de efectivo domina a los demás instrumentos de pago, independientemente del valor de la transacción (Bagnall et al., 2016).

Otro elemento que se atribuye a la mayor preferencia por el efectivo es su mayor aceptación, y es que este instrumento es el único que no está sujeto a costos de transacción (Whitesell, 1989). En contraste, la aceptación y el uso de tarjetas bancarias dependen de la estructura de tarifas que las rige, y cuyo manejo ha motivado la intervención de las autoridades regulatorias en 
algunos países europeos, en Australia y en los Estados Unidos (Koulayev et al., 2016). Los resultados de esas intervenciones varían de un lugar a otro, ya que dependen de la forma en la que los bancos trasladan los cambios a las tarifas (hacia los comerciantes o hacia los clientes bancarios) y de la forma en la que los comerciantes responden a las medidas de los bancos (trasladando total o parcialmente los cambios en las tarifas a sus clientes, o incentivando el uso de efectivo mediante descuentos). En este tema, la literatura ha señalado que los costos financieros que asumen los comerciantes al aceptar pagos con tarjeta explican el por qué usualmente los consumidores portan efectivo para realizar transacciones en lugares donde otros instrumentos de pago (como las tarjetas de crédito) no son aceptados (Dutta \& Weale, 2001; Bolt, Humphrey \& Uittenbogaard, 2005; Arango \& Taylor, 2009).

Los estudios empíricos sobre la preferencia del consumidor por instrumentos de pago pueden tener un enfoque micro cuando se basan en encuestas (Attanasio, Jappelli \& Guiso, 2002; Stix, 2004; Bounie \& Francois, 2006; Klee, 2008; Deutsche Bundesbank, 2009; Arango et al., 2011; O’Brien, 2014; Bagnall et al., 2016; Koulayev et al., 2016; Wakamori \& Welte, 2017) o un enfoque macro cuando se usan medidas relativas a los instrumentos de pago (Amromin \& Chakravorti, 2007). Los enfoques macro se relacionan con teorías de portafolio o con teorías de transacción. Las teorías de portafolio permiten modelar los motivos keynesianos precaución y especulación, los cuales se ajustan a definiciones de dinero como M2 y M3 que consideran la función de dinero como depósito de valor. Por otro lado, las teorías de transacción optan por definiciones de dinero como instrumento de pago (en el intercambio), como M1 y el efectivo (Ericsson, 1998). Si bien estos últimos podrían también satisfacer la función de depósito de valor, la diferencia entre las teorías de portafolio y de transacción radica en que M1 y el efectivo cuentan con alternativas ofrecidas por otros activos con un mejor perfil riesgo-retorno, los cuales hacen parte de agregados monetarios más amplios, como M2 y M3.

Los estudios empíricos sobre teorías de transacción han tratado el tema mediante enfoques sobre: i) tarjetas de crédito; ii) cajeros automáticos; iii) cajeros automáticos y transferencias electrónicas de fondos; iv) y transferencias electrónicas de fondos mediante tarjetas débito (Scholnick, et al. 2008). En este último grupo de estudios se ha considerado, adicionalmente, el costo de 
uso de los instrumentos de pago, su sustitución y los factores idiosincráticos. Este estudio se clasifica en ese último grupo, ya que considera el costo de usar tarjetas débito, la automatización de los servicios bancarios y sus efectos sobre las decisiones de pago de la población.

Las nuevas tecnologías de pago y su creciente participación en los servicios bancarios han generado un mayor uso de tarjetas débito y un menor uso de cheques en las transacciones de bajas cuantías. Esto se explica, por un lado, por el aumento en el número de canales transaccionales que ha permitido disminuir el costo real de usar instrumentos electrónicos como las tarjetas débito (Humphrey et al., 1996a; Humphrey, Kim \& Vale, 2001); pero, por otro lado, por el tiempo que tarda el canje de un cheque y su potencial riesgo de compensación -que depende de la suficiencia de fondos del girador- frente a la posibilidad de acreditar, mediante una compensación más rápida que los cheques, el valor de la transacción mediante tarjetas débito.

La sustitución entre instrumentos de pago ha motivado estudios que coinciden en afirmar que las tecnologías de pago electrónico han tenido un bajo impacto sobre el efectivo, pero no sobre los cheques (Drehmann et al., 2002; Humphrey, 2004). Para Humphrey (2004), el uso creciente de tarjetas débito en los Estados Unidos ha generado un desplazamiento de los cheques y un moderado reemplazo del efectivo. Amromin y Chakravorti (2009) coinciden con este resultado, pero señalan que el impacto de las tarjetas débito sobre la demanda de efectivo es exclusivo de billetes de baja denominación, ya que los de alta denominación son usados como depósito de valor y no para realizar transacciones.

El proceso de sustitución puede evidenciar similitudes entre países, pero la etapa del proceso en la que se encuentra cada país depende de la difusión de los canales de pago electrónico y de otros aspectos como los factores idiosincráticos (Snellman et al., 2001). Son precisamente estos factores los que permiten entender las diferencias que se presentan en la preferencia por el uso de efectivo en países que tienen un nivel similar progreso tecnológico, como el caso de Japón (16,3\%) y Reino Unido (3,4\%), lo cual se ha atribuido a las bajas tasas de inflación y crimen que se registran en el primer caso (Amromin \& Chakravorti, 2009). Algunos estudios han señalado que países con 
entornos seguros carecen de incentivos para promover el uso de instrumentos electrónicos de pago, mientras que en otros con una alta tasa de crimen y alto nivel de ingreso, como los Estados Unidos, presentan una mayor preferencia por cheques y tarjetas bancarias (Humphrey et al. 1996a, 1996b). Otros estudios han mostrado que las personas pueden preferir el efectivo debido al potencial riesgo de robo de sus tarjetas bancarias o al riesgo de suplantación por el robo de la información que éstas contienen -robo de identidad- (Kahn et al., 2005). En otras palabras, un bajo nivel de seguridad no necesariamente genera una sustitución de efectivo por tarjetas bancarias.

Los estudios que han revisado este tema para el caso de Colombia se han basado en enfoques tradicionales que han incluido las innovaciones financieras y la heterogeneidad de los agentes para profundizar en el entendimiento de la demanda de dinero. Gómez (1998) examinó el efecto que puede generar la velocidad de circulación del dinero en la cantidad demandada, incluyendo las innovaciones financieras medidas como una tendencia en el tiempo. Arango y González (2000), y Misas et al. (2003), estudiaron la heterogeneidad de los agentes en la demanda agregada de dinero, mediante especificaciones no lineales. Un trabajo que se relaciona de manera tangencial con esta corriente de la literatura es el de Gómez-González, Jaramillo-Escobar y Meisel-Roca (2016), en el que se estudian los determinantes de la demanda de cuentas de depósito y crédito.

\section{Pagos con efectivo y tarjetas débito: un enfoque de flujo}

Estudios previos sobre la demanda de efectivo con motivo transacción han utilizado medidas stock y flujo del efectivo. Las medidas stock hacen referencia al número de transacciones, definido mediante indicadores de efectivo, tales como la razón efectivo a producto interno bruto (PIB) per cápita (Humphrey et al. 1996a; Drehmann et al., 2002; Amromin \& Chakravorti 2009). La posible relación inversa que puede existir entre esas medidas de efectivo y la velocidad del dinero (i.e. un aumento en el número de ATM podría reducir el stock de dinero real per cápita, pero al mismo tiempo aumentar los pagos per cápita con efectivo) ha hecho que sean cada vez más cuestionadas y menos utilizadas en estudios empíricos. Las medidas flujo, por su parte, 
representan los gastos en efectivo mediante los retiros en ATM o datos de encuestas como los diarios de pago. En este documento se estudian las preferencias por efectivo y tarjetas débito, siguiendo un enfoque macro y haciendo uso de una medida flujo del efectivo (i.e. el valor total de los retiros realizados con tarjetas débito).

La cuantificación de las preferencias de pago se limita a las personas que tienen cuentas de depósito (corrientes y de ahorros), puesto que son los únicos que pueden escoger entre efectivo y otros instrumentos de pago. El monto total de efectivo del que dispone este segmento de la población se mide como el valor total de los retiros con tarjetas débito, en pesos de $2008^{2}$. Se excluyen los avances con tarjetas de crédito y créditos rotativos porque el propósito principal del documento es estudiar los instrumentos de pago que provienen del mismo medio de pago: depósitos en cuentas corrientes y de ahorros. La pregunta que surge en este punto es ¿qué tan representativa es esta medida del efectivo total que circula en la economía? Siguiendo el trabajo de Humphrey (2004), se calcula el efectivo total que usa la población descontando al consumo privado el valor de los pagos realizados con tarjetas bancarias y cheques, y usando datos anuales sobre el consumo de los hogares de cuentas nacionales, del valor del consumo con tarjetas de crédito y débito y del valor de los cheques compensados en el Cedec ${ }^{3}$.

$$
\left(\begin{array}{c}
\text { Pagos en } \\
\text { efectivo }
\end{array}\right)=\left(\begin{array}{c}
\text { Consumo } \\
\text { privado }
\end{array}\right)-\left(\begin{array}{c}
\text { Consumo } \\
\text { pagado con } \\
\text { tarjetas } \\
\text { bancarias }
\end{array}\right)-\left(\begin{array}{c}
\text { Consumo } \\
\text { pagado con } \\
\text { cheques }
\end{array}\right) \text {. }
$$

De los datos obtenidos, se observa que los retiros con tarjetas débito representan $40 \%$ del efectivo total usado en la economía.

Al igual que en el trabajo de Amromin y Chakravorti (2007), la forma funcional para modelar el uso de tarjetas débito es logarítmica, y depende de los canales transaccionales (ATM y datáfonos, por habitante) y de la tasa de interés de corto plazo. Como se observa en la ecuación (2), los retiros de efectivo per cápita con tarjetas débito (RT_EFECTIVO) dependen del número per cápita de compras con tarjetas débito (NC_TDEBITO), y viceversa (ecuación (3)).

2 El valor de los retiros se deflactó con el índice de precios al consumidor.

3 Cedec es el sistema de compensación electrónica del Banco de la República donde se compensan cheques y otros instrumentos de pago entre bancos. 
Martínez: El uso de efectivo y tarjetas débito como instrumentos de pago en Colombia

De tal forma, se define un sistema de ecuaciones que incluye, adicionalmente, la producción y algunos factores idiosincráticos (gravamen a los movimientos financieros -GMF-, informalidad y crimen).

$$
\begin{aligned}
\text { RT_EFECTIVO }_{t} & =\alpha_{0}+\alpha_{1} \text { ATMS }_{t}+\alpha_{2} \text { Datáfonos }_{t}+\alpha 3 \text { T.I }_{t} \\
& +\alpha_{4} \mathrm{GMF}_{t}+\alpha_{5} \text { Producción }_{t}+\alpha_{6} \text { Informalidad }_{t} \\
& +\alpha_{7} \text { Crimen }_{t}+\alpha_{8} \text { NC_TDEBITO }_{t}+u_{t},
\end{aligned}
$$

$$
\begin{aligned}
\text { NC_TDEBITO }_{t} & =\beta_{0}+\beta_{1} \text { ATMS }_{t}+\beta_{2} \text { Datáfonos }_{t}+\beta 3 \text { T.I }_{t} \\
& +\beta_{4} \text { Cheques }_{t}+\beta_{5} \text { Producción }_{t}+\beta_{6} \text { Informalidad }_{t} \\
& +\beta_{7} \text { Crimen }_{t}+\beta_{8} \text { RT_EFECTIVO }_{t}+v_{t}
\end{aligned}
$$

Los parámetros de las variables explicativas y el uso de tarjetas débito y efectivo se relacionan de manera lineal. Los residuos de cada ecuación $\left(u_{t}, v_{t}\right)$ se asumen no correlacionados entre sí.

Según Amromin y Chakravorti (2007), en ausencia de datos sobre los costos de usar tarjetas débito para realizar pagos (como sucede para Colombia), es apropiado utilizar cifras sobre canales transaccionales, suponiendo que a medida que se incrementa su número están disminuyendo los costos de usar los instrumentos de pago relacionados con estos canales ${ }^{4}$. Esta particularidad hace que el sistema de ecuaciones no se pueda considerar un modelo de demanda de pagos estructural, ya que no permite calcular elasticidades precio de usar los instrumentos de pago. El único parámetro que está capturando una elasticidad precio es el de la tasa de interés, por representar el costo de oportunidad que los usuarios bancarios asumen por mantener saldos reales ${ }^{5}$.

4 Existen otros parámetros (precios) que intervienen en el uso de tarjetas débito, tales como las cuotas de manejo que pagan los tarjetahabientes (mercado de emisión), las comisiones por adquiriencia cobradas a comerciantes (mercado de adquiriencia) y la tarifa interbancaria de intercambio que opera entre bancos adquirientes y emisores. Lamentablemente no se cuenta con información que permita su cuantificación en este estudio.

5 Los costos de los servicios que las tarjetas débito ofrecen a sus usuarios incluyen costos fijos (cuotas de manejo) y costos variables relativos al número de retiros que realizan. 


\section{Datos}

El sistema de ecuaciones se estima con información mensual desde abril de 2002 hasta abril de 2012, lo que corresponde a 121 observaciones. Como se mencionó anteriormente, el uso de efectivo se mide como el valor total de los retiros con tarjetas débito, a precios constantes de 2008. El uso de las tarjetas débito se mide por el número per cápita de las compras realizadas con este instrumento de pago, para el cual las cifras revelan un creciente uso, dado por un volumen de compras que pasó de 204.490 en 2002, a 1’164.169 en 2011.

El modelo descrito por las ecuaciones (2) y (3) tiene como propósito medir la elasticidad de la demanda transaccional de efectivo y tarjetas débito frente a cambios porcentuales en sus determinantes tradicionales (producción y tasa de interés) y no tradicionales (infraestructura de pagos y factores idiosincráticos).

En el primer grupo de variables explicativas están el indice de producción industrial y la tasa de interés nominal, obtenidas del Departamento Administrativo Nacional de Estadística (DANE) y Banco de la República, respectivamente. El índice de producción industrial, se incluye como proxy de la producción total (PIB), la cual solo está disponible trimestralmente. La producción industrial se trabaja en términos reales (base $2008=100$ ) para que sea comparable con las medidas de uso de efectivo y tarjetas débito. La tasa de interés nominal (efectiva anual), corresponde a la que los bancos reconocen a sus clientes por el dinero en sus cuentas de depósito. Esta variable tiene como propósito cuantificar el costo que para los agentes representa portar efectivo, e incluye el rendimiento que este redituaría en una cuenta de depósitos (costo de oportunidad) y los cambios en el nivel de precios, ya que el efectivo no representa ninguna cobertura contra presiones inflacionarias. Otras tasas de interés como las de los depósitos a término fijo (DTF) y las de certificados de depósitos a término (CDT) no se consideran determinantes de la demanda transaccional de efectivo, por ser de mediano y largo plazo.

Para estudiar la infraestructura de pagos se utilizan datos sobre los canales transaccionales que permiten realizar pagos y retiros de efectivo (ATM), y que además facilitan el traslado de dinero de las cuentas de depósitos de compra- 
Martínez: El uso de efectivo y tarjetas débito como instrumentos de pago en Colombia

dores hacia las de comerciantes (datáfonos). Otros canales, como las oficinas bancarias, no se incluyen, puesto que sus estadísticas, en número de unidades, solo están disponibles trimestralmente. El número de ATM por cada cien mil habitantes pasó de 13 en 2002 a 23 en 2011, mientras que el número promedio de retiros mensuales (por cada cien mil habitantes) pasó de 48.470 a 82.050 en los mismos años. Los datos sobre datáfonos provienen de una de las franquicias de tarjetas bancarias que operan en el país, y representan alrededor de $50 \%$ de los dispositivos en funcionamiento. No obstante, se considera que esta información es apropiada porque, por un lado, la serie de datos exhibe tendencia creciente, similar a la de los establecimientos comerciales afiliados a las franquicias de tarjetas (Gráfico 1) y, por otro lado, da cuenta de la aceptación de las diferentes tarjetas bancarias, independientemente de su franquicia. La tendencia creciente que presenta el número de datáfonos para el periodo de estudio sugiere una creciente disponibilidad de tecnología para efectuar pagos electrónicos.

Gráfico 1. Datáfonos y establecimientos comerciales, 2002-2011

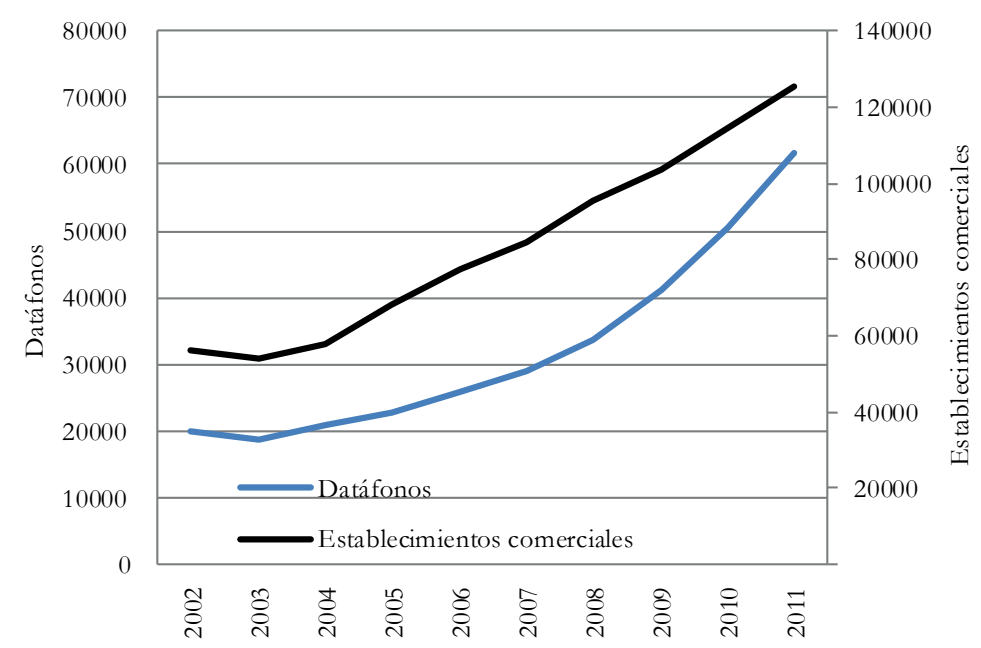

Nota: información obtenida bajo petición y con acuerdo de confidencialidad. Fuente: elaboración propia con base en información de redes y Asobancaria.

Como factores idiosincráticos se incluyen el GMF, un indicador de inseguridad y uno de informalidad. 
El GMF se introdujo en la economía en noviembre de 1998 como un impuesto transitorio, con el propósito de garantizar la estabilidad del sistema financiero. Por su fácil recaudo su permanencia se prolongó indefinidamente, y su tasa pasó de $0,2 \%$ a $0,3 \%$ en diciembre de 2000 , y a $0,4 \%$ desde diciembre de $2003^{6}$. Algunos autores como Coelho, Ebrill y Summers (2001) consideran que este impuesto es la principal razón del aumento en la desintermediación financiera, ya que motiva en los agentes el uso de estrategias para eludir su pago, tales como evitar que el efectivo pase a través del sistema financiero, reducir la rotación de las cuentas de depósitos y realizar múltiples endosos de cheques.

Como indicador de inseguridad, se calcula el número de homicidios per cápita (crimen $p c p$ ), usando información registrada por la Policía Nacional sobre este crimen y cifras de población reportadas por del DANE. Esta variable se incluye con el fin de establecer si existe una sustitución entre instrumentos de pago a medida que disminuye la seguridad del entorno en el que la población realiza sus transacciones. No se incluyeron otros indicadores de seguridad porque el homicidio es el delito que representa mayor gravedad para la población, pero también porque las personas tienden a no denunciar otros delitos, como el hurto a personas, lo que puede llevar a que se subestime la percepción de seguridad.

Otro factor idiosincrático es la economía informal, que usualmente se relaciona con las ventas callejeras, el contrabando y la producción y el tráfico de estupefacientes; todas estas actividades hacen uso intensivo del efectivo, pues buscan evitar la trazabilidad. Por la naturaleza de estas actividades no se dispone de cifras que permitan su cuantificación específica; sin embargo, se propone una medición indirecta de al menos uno de estos aspectos, en este caso, del comercio informal. Para esto, se usa la tasa de subempleo subjetivo reportada por el DANE como proxy de informalidad, la cual se explica por insuficiencia de horas trabajadas (inferior a la jornada semanal) y por condiciones de empleo inadecuado. Aunque se reconocen las limitaciones de esta variable para cuantificar el comercio informal, medidas alternativas como el porcentaje de población que cotiza seguridad social (salud y pensión) no solo

$\overline{6}$ Decreto 2331 de 1998, Ley 633 de 2000 y Ley 863 de diciembre de 2003. 
Martínez: El uso de efectivo y tarjetas débito como instrumentos de pago en Colombia

tienen una frecuencia distinta (trimestral) a la que se usa para las demás variables, sino que tampoco están disponibles para todo el período de estudio. La proxy de informalidad utilizada (tasa de subempleo subjetivo) presenta una alta coincidencia con datos anuales de empleo en el sector informal (su coeficiente de correlación es 0,91).

Además de estas variables explicativas, se incluyen los cheques por ser los que seguían en importancia al efectivo antes que se diera la popularización de las tarjetas débito a finales de 1990. Como proxy del total de cheques girados se utiliza el número de cheques compensados en la primera sesión del Cedec, excluyendo aquellos cuyo valor superan el millón de pesos para no contabilizar los que fueron girados para la compra de bienes durables. Para el periodo de estudio se observa un descenso en el número de cheques y un aumento en el de tarjetas débito, que coinciden con el surgimiento de las transferencias electrónicas de fondos entre los usuarios de servicios bancarios (Gráfico 2).

Gráfico 2. Transacciones con tarjetas débito y cheques

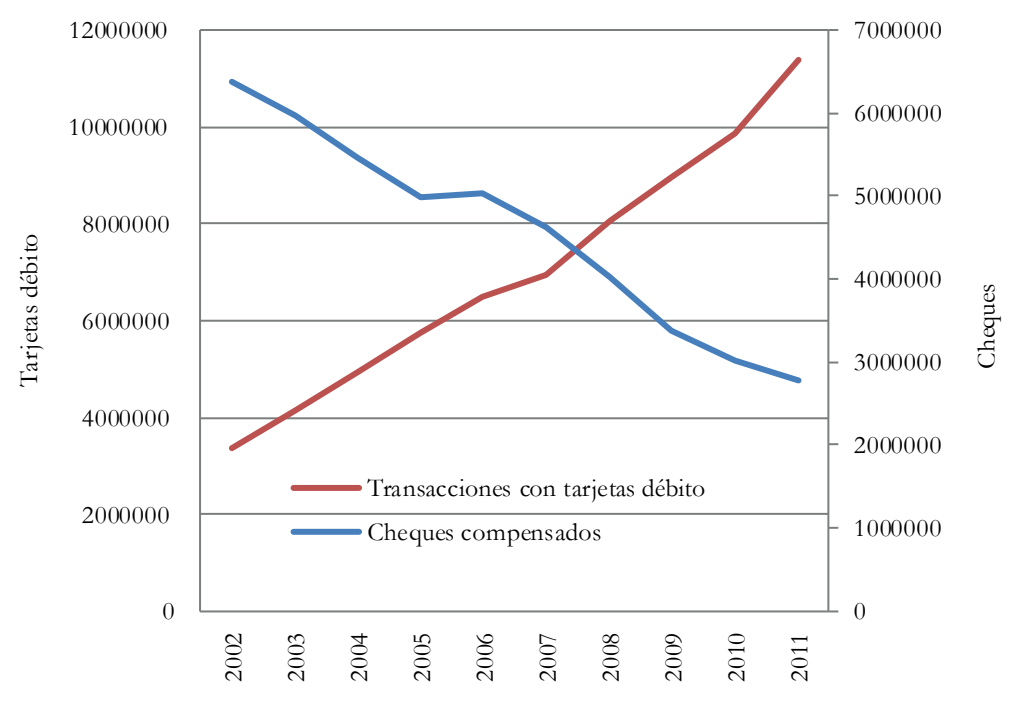

Nota: información obtenida bajo petición y con acuerdo de confidencialidad. Fuente: elaboración propia con base en información de Asobancaria, Superfinanciera, Banco de la República y DANE. 


\section{Resultados}

Las variables dependientes (retiros con tarjetas débito y número de compras con tarjetas débito, per cápita) están atadas a los mismos medios de pago (depósitos en cuentas corrientes y de ahorros), lo cual puede sugerir una determinación conjunta de las mismas o endogeneidad. Los modelos de ecuaciones simultáneas hacen un tratamiento apropiado a este problema, ya que permiten realizar una estimación más precisa de los parámetros de cada ecuación.

La presencia de endogeneidad en el sistema de ecuaciones se contrastó mediante la prueba Durbin-Wu-Hausman en estimaciones por 2SLS (MCO en dos etapas) de tres especificaciones (ver Tabla 1). El modelo 1 excluye las variables idiosincráticas de informalidad y crimen, el modelo 2 excluye la variable crimen y el modelo 3 contiene todas las variables.

Tabla 1. Prueba Durbin-Wu-Hausman

\begin{tabular}{cccc}
\hline & & $\begin{array}{l}\text { Log(Retiros de efectivo con } \\
\text { tarjetas débito, pcp) }\end{array}$ & $\begin{array}{l}\text { Log(Número de compras con } \\
\text { tarjeta débito, pcp) }\end{array}$ \\
\hline \multirow{2}{*}{ Modelo 1 } & $\chi^{2}(1)$ & 19,38 & 12,94 \\
& Valor $\mathrm{p}$ & 0,000 & 0,000 \\
\hline \multirow{2}{*}{ Modelo 2 } & $\chi^{2}(1)$ & 15,93 & 16,72 \\
& Valor $\mathrm{p}$ & 0,000 & 0,000 \\
\hline \multirow{2}{*}{ Modelo 3 } & $\chi^{2}(1)$ & 22,12 & 19,20 \\
& Valor $\mathrm{p}$ & 0,000 & 0,000 \\
\hline
\end{tabular}

Fuente: elaboración propia.

Los resultados indican que las variables dependientes retiros y numero compras con tarjetas débito son endógenas. Este problema puede atribuirse a que ambas variables dependen del mismo medio de pago, es decir, de depósitos en cuentas corrientes y de ahorros. Si se ignora el problema de endogeneidad y se realizan estimaciones independientes de las ecuaciones se pueden obtener parámetros sesgados e inconsistentes ${ }^{7}$. En la Tabla 2 se reportan resultados

7 En sistemas de ecuaciones de este tipo donde cada ecuación está exactamente identificada, los métodos de estimación con información limitada y con información completa generan los mismos parámetros, pero éste último genera estimadores más eficientes, es robusto a problemas de no normalidad y no requiere de errores esféricos. 
Martínez: El uso de efectivo y tarjetas débito como instrumentos de pago en Colombia

de estimaciones por 3SLS (MCO en tres etapas). Los resultados de la prueba Jarque-Bera multivariada sugieren ausencia de problemas de no normalidad ${ }^{8}$.

La automatización de los servicios bancarios, compuesta por ATM y datáfonos, puso a disposición de los clientes bancarios canales transaccionales que compiten entre sí, los primeros como proveedores de efectivo y para realizar pagos, y los segundos como dispositivos para realizar compras y efectuar pagos. Para los $A T M$, se observa el esperado signo positivo y significativo sobre los retiros con tarjetas débito, mientras que para los datáfonos se observa el efecto contrario. Según estos resultados, existe algún grado de sustitución entre el efectivo y las tarjetas débito en establecimientos del comercio formal y que cuentan con tecnologías de pago modernas. Los modelos para tarjetas débito exhiben una asociación negativa con los ATM y positiva con los datáfonos que podrían sugerir, nuevamente, ese efecto sustitución. Este resultado señala, por un lado, un mayor uso de las tarjetas débito y, por otro lado, una mayor aceptación de las mismas por parte de los comerciantes, lo que concuerda con la reducción en términos reales que en los últimos años han experimentado las tarifas que los bancos cobran a comerciantes y usuarios de tarjetas. Este resultado coincide con lo expuesto por Bardey y Meléndez (2012), quienes describen las modificaciones a la tarifa de intercambio (i.e. pago que el banco adquiriente hace al banco emisor por cada transacción en la que participa) que la Superintendencia de Industria y Comercio sugirió en 2006 y en 2008 a las redes y a los bancos participantes del mercado de tarjetas, y que consistieron en atar la estructura de tarifas a modelos de costos.

Otro determinante esencial son los cheques, pues su efecto sobre las compras con tarjetas débito es negativo y significativo en las tres estimaciones. Este resultado coincide con los de Drehmann et al. (2002) y Humphrey (2004), y el efecto sustitución que identifica se considera evidencia del desplazamiento que la popularización de las tarjetas débito ha ocasionado a los cheques, en lo que se refiere a compras de bajos montos.

Con respecto a los factores idiosincráticos, se observa una relación negativa y significativa entre el GMF y el efectivo, que sugiere una disminución de los retiros con tarjetas débito a causa de este impuesto. Según los argumentos de

8 Esta prueba de normalidad usa el método de ortogonalización de Doornik-Hansen. 


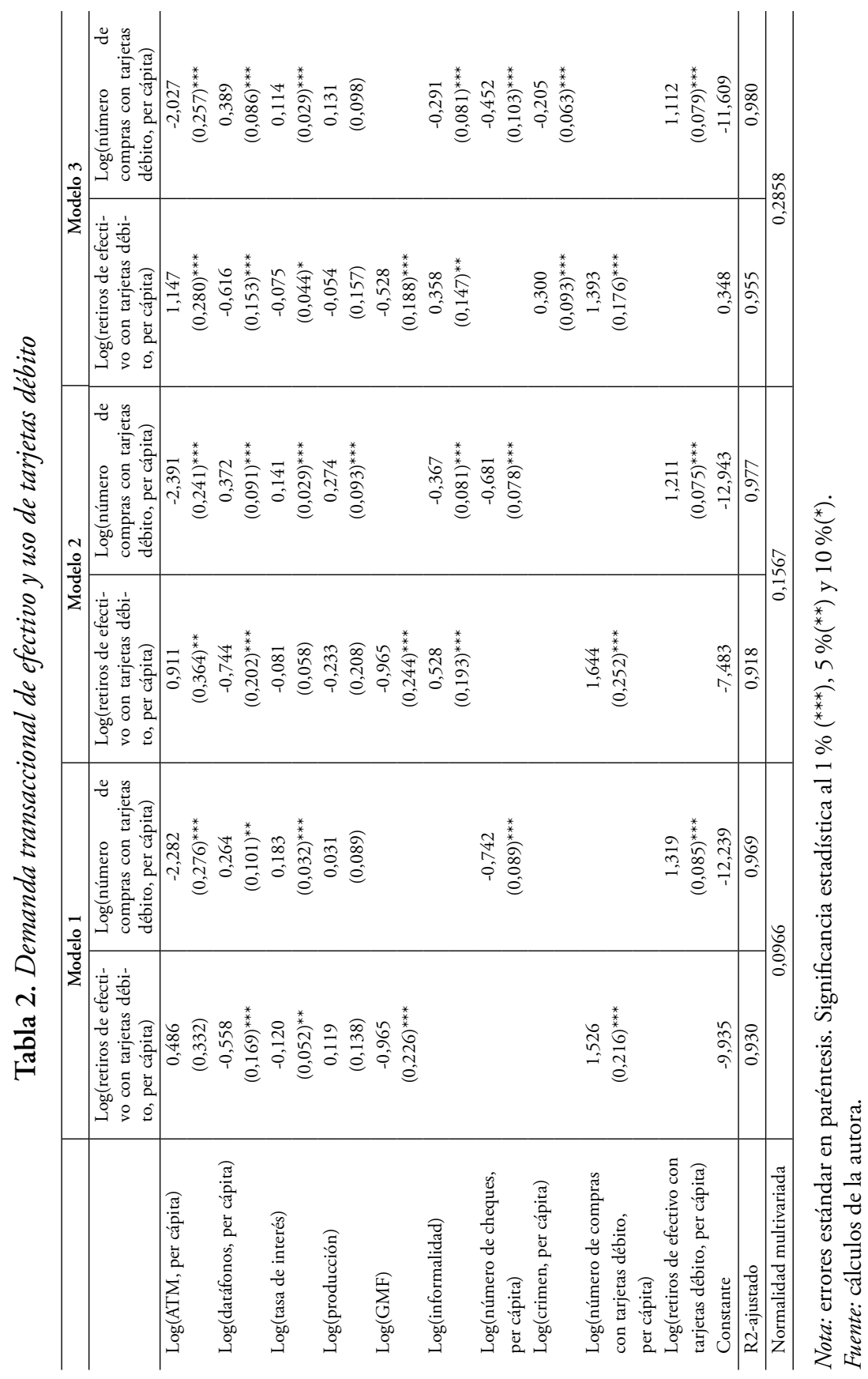


Martínez: El uso de efectivo y tarjetas débito como instrumentos de pago en Colombia

Coelho et al. (2001), un efecto negativo de este impuesto sobre el efectivo se puede atribuir a que la población trata de eludir su pago (total o parcialmente) disminuyendo la rotación de las cuentas de depósito.

La variable crimen exhibe un signo positivo sobre los retiros de efectivo y negativo sobre el número de compras con tarjetas débito, lo que coincide con la premisa de Kahn et al. (2005) sobre el papel del efectivo al atenuar el riesgo de robo de identidad a los usuarios de tarjetas de crédito (ver modelo 3 de la Tabla 2). Este resultado indica que las personas con cuentas de depósito pueden disminuir el uso de tarjetas débito para evitar ser víctimas de la inseguridad y, en consecuencia, deciden pagar con efectivo por temor a que clonen o cambien su tarjeta débito en establecimientos comerciales no conocidos ${ }^{9}$. Otra explicación a este resultado puede ser que las personas deciden no portar sus tarjetas débito por temor al "paseo millonario", que es una modalidad de hurto en Colombia cuya duración depende del tiempo requerido por los criminales para sustraer el valor máximo permitido por retiros diarios ${ }^{10}$.

La variable de informalidad exhibe también el esperado efecto positivo en la ecuación de retiros y efecto negativo en la de tarjetas débito, lo cual indica que, a medida que aumenta la dinámica de actividades como el comercio informal, la población aumentará el uso de efectivo y disminuirá el de tarjetas débito. Estos resultados se explican también por la ausencia de trazabilidad y la mayor aceptabilidad que el efectivo confiere a sus usuarios.

De los determinantes tradicionales incluidos, solo la tasa de interés resultó significativa, además de exhibir la relación esperada con ambos instrumentos de pago: negativa para retiros de efectivo y positiva para compras con tarjetas débito. Según el modelo 3 (ver Tabla 2), este resultado revela que, ante un incremento de $10 \%$ en la tasa de interés, los retiros de efectivo disminuirán en $0,75 \%$, mientras que las compras con tarjetas débito aumentarán en 1,15\%. Ambos resultados indican un comportamiento racional por parte de los clientes

9 Si bien sería interesante incluir los delitos informáticos solo existen cifras desde enero de 2009, que fue cuando se tipificó esta conducta como delito contra el patrimonio económico (Ley 1273 de 2009).

10 Según la Policía Nacional, el "paseo millonario" implica penas por hurto calificado y secuestro extorsivo. Desafortunadamente, no existen estadísticas sobre este tipo de delito. 
bancarios, según el cual, a medida que aumentan los retornos sobre los saldos en sus cuentas de depósito, ellos preferirán disminuir el valor de sus retiros.

En conjunto, los resultados sugieren que el efectivo está lejos de desaparecer, aun cuando las alternativas de pago electrónicas ofrecen cada vez más y mejores soluciones tecnológicas para la población usuaria de servicios bancarios. Este resultado es particular de transacciones de bajas cuantías y coincide con los obtenidos para países desarrollados (Drehman et al., 2002; Amromin \& Chakravorti, 2009; Bagnall et al., 2016). Estudios previos, como el de Whitesell (1989), han relacionado la preferencia por efectivo en pagos de bajo valor con el bajo costo de oportunidad que implica su uso, frente al costo fijo que implica usar instrumentos de pago alternativos.

\section{Conclusiones}

El desarrollo de la infraestructura de pagos y canales transaccionales ha puesto a disposición de los clientes bancarios más y mejores soluciones para realizar sus pagos a otras personas, al sector comercial y de servicios. En la última década, estos cambios han avanzado rápidamente, pero como se observa en este estudio, para el caso colombiano, sus efectos en las preferencias de pago de la población parecen avanzar a una menor velocidad.

En este documento se estudian las preferencias por instrumentos de pago de las personas que tienen cuentas de depósito, ya que estas son las únicas que pueden elegir realizar, de manera presencial, pagos con efectivo, tarjetas débito o cheques. Al delimitar la unidad de análisis a ese segmento poblacional se supone implícitamente que, en sus pagos en efectivo, tarjeta débito y cheques, hacen uso exclusivo de los saldos depositados en sus cuentas corrientes y de ahorro. De tal forma, el uso de la tarjeta débito se cuantifica mediante el número de pagos realizados con este instrumento, mientras que el de efectivo se mide por el valor de los retiros realizados con tarjetas débito. El uso de cheques se mide de manera indirecta, a partir de información suministrada por el Cedec. Las mediciones se concentran en pagos de bajo valor y en compras al por menor de bienes no durables. 
Martínez: El uso de efectivo y tarjetas débito como instrumentos de pago en Colombia

La infraestructura de pagos exhibe los efectos esperados, tanto para pagos con tarjetas débito como para pagos con efectivo. En el caso de los datáfonos, se observa el efecto positivo esperado sobre el uso de tarjetas débito y negativo sobre el uso de efectivo, mientras que en el caso de los cajeros automáticos se corrobora el efecto contrario, el cual favorece los pagos con efectivo. No obstante, en ambos casos (uso de efectivo y tarjetas débito) los coeficientes estimados para los ATM son mayores que los obtenidos para datáfonos, lo cual sugiere una mayor aceptación del efectivo. Este resultado se puede atribuir al carácter anónimo que el efectivo confiere a sus usuarios, al hecho de que reduce el riesgo de clonación y robo de tarjetas, pero también a otros factores no medidos directamente, como los precios relativos de usar otros instrumentos de pago.

Las decisiones de la población sobre cuál instrumento usan para pagar dependen también de la idiosincrasia de sus habitantes. En este tema, se observa cómo, a medida que aumenta el crimen y la informalidad, se genera un mayor uso de efectivo y un menor uso de tarjetas débito. Caso contrario sucede con el GMF, ya que, según los resultados, las personas tienden a disminuir sus retiros de efectivo a causa de este impuesto, por considerarlo un sobrecosto a las tarifas por servicios bancarios. Los resultados también señalan un desplazamiento de los cheques por parte de las tarjetas débito (lo cual coincide con las hipótesis planteadas inicialmente) debido a aspectos como el costo que representa para la persona que lo gira, el tiempo de canje, el riesgo de compensación y el cargo adicional que implica el GMF.

En síntesis, aun cuando los resultados sugieren algún grado de sustitución entre el efectivo y la tarjeta débito en las compras al por menor de bienes no durables, tal sustitución no es perfecta.

Como extensiones a este tema, se plantea la medición de los impactos que podrían generar los corresponsales no bancarios sobre el uso de los instrumentos de pago, para evaluar el alcance de esta política de inclusión financiera sobre las preferencias de pago de la población ${ }^{11}$. Asimismo, se considera in-

11 Estos establecimientos no crediticios son canales transaccionales alternativos a los ATM y a las sucursales bancarias, contratados por los bancos para prestar servicios en zonas con limitada presencia bancaria. 
teresante realizar estudios usando datos de encuestas, para caracterizar los tipos de agentes y sus preferencias por instrumentos al momento de pagar. Finalmente, otra extensión interesante sería estudiar los pagos y las compras realizadas en canales no presenciales como el internet, y los efectos que los delitos informáticos podrían generar sobre ese tipo de transacciones.

\section{Referencias}

Amromin, Gene \& Chakravorti, Sujit (2007). "Debit Cards and Cash usage: A Cross Country Analysis”, Working paper 2007-04. Federal Reserve Bank of Chicago.

Amromin, Gene \& Chakravorti, Sujit (2009). "Whither loose change? The Diminishing demand for small denomination currency", Journal of Money, Credit and Banking, Vol. 41, No. 2/3, pp. 315-335.

Arango, Luis \& González, Andrés (2000). "A nonlinear specification of demand for cash in Colombia", Money Affairs, Vol. XIII, No. 2, pp. 207-226.

Arango, Carlos \& Taylor, Varya (2009). "The Role of Convenience and Risk in Consumers' Means of Payment", Discussion Paper, No. 2009-8. Bank of Canada.

Arango, Carlos; Huynh, Kim \& Sabetti, Leonard (2011). "How do you pay? The role of incentives at the point of sale", Working Paper, No. 1386. European Central Bank.

Attanasio, Orazio; Jappelli, Tullio \& Guiso, Luigi (2002). “The Demand for Money, Financial Innovation, and the Welfare Cost of Inflation: An analysis with Household data", Journal of Political Economy, Vol. 110, No. 2, pp. 317-351.

Bagnall, John; Bounie, David; Huynh, Kim; Kosse, Anneke; Scmidt, Tobias; Schuch, Scott \& Stix, Helmut (2016). "Consumer Cash Usage: A cross-Country Comparison with Payment Diary Survey Data", International Journal of Central Banking, Vol. 12, No. 4, pp. 1-61. 
Martínez: El uso de efectivo y tarjetas débito como instrumentos de pago en Colombia

Bardey, David \& Meléndez, Marcela (2012). "La economía de los mercados de dos lados: aplicación al análisis de las tarjetas de pago en Colombia", Documentos CEDE, No. 27. Universidad de los Andes.

Bolt, Wilko; Humphrey, David \& Uittenbogand, Roland (2005). "The Effect of Transaction Pricing on the Adoption of Electronic Payments: A Cross-Country Comparison", Working Paper, No. 05-28. Federal Reserve.

Bounie, David \& Francois, Abel (2006). "Cash, Check or Bank Card?”, Working Papers in Economics and Social Sciences, No. ESS-06-05. Telecom Paris Tech.

Coelho, Isaias; Ebrill, Liam \& Summers, Victoria (2001). "Bank debit taxes in Latin America: An Analysis of Recent Trends", IMF Working paper, No. 67. International Monetary Fund.

Decreto 233 I De 1998. Ministerio de Hacienda y Crédito Público, Diario Oficial No. 43.430, noviembre 16.

Deutsche Bundesbank (2009). "Choosing between payment instruments and their usage: a microeconometric analysis on the consumer level", In Payment behaviour in Germany. An empirical study of the selection and utilization of payment instruments in the Federal Republic of Germany (pp. 68-78). Disponible en: https://www.bundesbank.de/Redaktion/EN/Downloads/Publications/Studies/payment_ behaviour_in_germany_in_2009.html (enero 2013).

Dremmann, Mathias; Goodhart, Charles \& Krueger, Malte (2002). "The Challenges Facing Currency Usage: Will the Traditional Transaction Medium Be Able to Resist Competition from the New Technologies?”, Economic Policy, Vol. 17, No. 34, pp. 193-227.

Dutta, Jayasri \& Weale, Martin (2001). "Consumption and the Means of Payment: An Empirical Analysis for the United Kingdom”, Economica, Vol. 68, No. 271, pp. 293-316.

Ericsson, Neil (1998). "Empirical modeling of money demand", Empirical Economics, Vol. 23, No. 3, pp. 295-315. 
Gómez, Javier (1998). "La Demanda de dinero en Colombia”, Borradores de Economía, No. 101. Banco de la República, Colombia.

Gómez-González, José; Jaramillo-Echeverri, Juliana \& Meisel-Roca, Adolfo (2016). "El Uso de Efectivo y Tarjetas Débito y Crédito en Colombia”, Borradores de Economía, No. 950. Banco de la República, Colombia.

Hernández, Antonio (2009). "Banca móvil y Bancarización de los pobres: Implicaciones macroeconómicas”, USAID, Programa MIDAS. Mimeo.

Humphrey, David; Pulley, Lawrence \& Vesala, Jukka (1996a). “Cash, Paper and Electronic Payments: A Cross country analysis", Journal of Money, Credit and Banking, Vol. 28, No. 4, pp. 914-939.

Humphrey, David; Sato, Setsuya; Tsurumi, Masayoshi \& Vesala, Jukka (1996b). "The Evolution of payments in Europe, Japan and the United States: Lessons for Emerging Economies", World Bank Working Paper, No. 1676, pp. 1-44.

Humphrey, David; Kim, Moshe \& Vale, Bent (2001). "Realizing the gains from electronic payments: Costs, pricing and payment choice", Journal of Money, Credit and Banking, Vol. 33, No. 2, pp. 216-234.

Humphrey, David (2004). "Replacement of cash by cards in U.S consumer payments", Journal of Economic and Business, Vol. 56, No. 3, pp. 211225.

Kahn, Charles; Mcandrews James \& Roberds, William (2005). "Money is Privacy”, International Economic Review, Vol. 46, No. 2, pp. 377-399.

KLEE, Elizabeth (2008). "How people pay: Evidence from grocery store data", Journal of Monetary Economics, Vol. 55, No. 3, pp. 526-541.

Koulayev, Sergei; Rysman, Marc; Schuh, Scott \& Stavinss, Joanna (2016). "Explaining adoption and use of payment instruments by US consumers", RAND Journal of Economics, Vol. 47, No. 2, pp. 239-325.

LeY 633 DE 2000. Diario Oficial No. 44.275, diciembre 29. 
Martínez: El uso de efectivo y tarjetas débito como instrumentos de pago en Colombia

LeY 863 DE 2003. Diario Oficial No. 45.415, diciembre 29.

Misas, Martha; López, Enrique; Arango, Carlos \& Hernández, Juan Nicolás (2003). "La Demanda de Efectivo en Colombia: Una caja negra a la luz de las redes neuronales", Borradores de Economía, No. 268. Banco de la República, Colombia.

O'Brien, Shaun (2014). "Consumer Preferences and the Use of Cash: Evidence from the Diary of Consumer Payments Choice", Working Paper. Federal Reserve Bank of San Francisco. Disponible en: https://www.frbsf.org/cash/files/OBrien-2014-Consumer-Preferencesand-the-Use-of-Cash-Evidence-from-the-Diary-of-ConsumerPayments-Choice.pdf (agosto 2014).

Rambure, Dominique \& Nacamuli, Alec (2008). "Payment Instruments". En: Payment Systems: from Salt Mines to Board Room (pp. 23-42). United Kindom: Palgrave MacMillan.

Scholnick, Barry; Massoud, Nadia; Saunders, Anthony; Carbo, Santiago \& Rodríguez, Francisco (2008). "The economics of credit cards, debit cards and ATMs: A survey and some new evidence", Journal of Banking and Finance, Vol. 32, No. 8, pp. 1468-1483.

Snellman, Jussi; Vesala, Jukka \& Humphrey, David (2001). "Substitution of non-cash payment instruments for cash in Europe", Journal of Financial Services Research, Vol. 19, No. 2-3, pp. 131-145.

Stix, Helmut (2004). "How Do Debit Cards Affect Cash Demand? Survey Data Evidence”, Empirica, Vol. 31, No. 2, pp. 93-115.

Uribe, José; Ortiz, Carlos \& García, Gustavo (2008). “Informalidad y subempleo en Colombia: dos caras de la misma moneda", Cuadernos de Administración, Vol. 21, No. 37, pp. 211-241.

Wakamori, Naoki \& Welte, Angelika (2017). "Why do Shoppers Use Cash? Evidence from shopping Diary Data", Journal of Money, Credit and Banking, Vol. 49, No. 1, pp. 115-169. 
Whitesell, William (1989). "The demand for currency versus debitable accounts", Journal of Money, Credit and Banking, Vol. 21, No. 2, pp. 246251. 